Barry A. Finegan mB FFarcs(I), M. David Bukht MB FFARCS

\title{
Venous pressures in the isolated upper limb during saline injection
}

Venous pressure changes were assessed in the antecubital vein of an isolated arm during saline injection via an intravenous site on the dorsum of the hand. Although leak of contrast medium has been radiologically demonstrated in these circumstances, the compliance of the venows system of the isolated and exsanguinated limb has not been investigated. In five male and five female wolunteers, after exsanguination and isolation of the upper limb, volumes of $40 \mathrm{ml}$ or $60 \mathrm{ml}$ of physiological saline were injected manually. The resultant pressure changes in the antecubital vein were continuously recorded during and afier injection, until a steady state was reached.

Although peak pressures as high as $190 \mathrm{mmHg}$ were noted early in the injection, venous pressures tended to plateau before the injection was complete and remained constant until the end of the injection. Resting venous pressures of 20 to $50 \mathrm{mmHg}$ at the end of infusion occurred in all subjects. At no time did venous pressure approcch cuff pressure. The mechanism of leak with an intact tourniquet is not explained, in our study. by the development of venous pressure in excess of tourniquet pressure in the isolated limb.

\section{Key words}

ANAESTHETIC TECHNIQUES: regional, intravenous regional anaesthesia.

From the Department of Anaesthesia, Foothills Hospital at the University of Calgary, Calgary, Alberta.

Address correspondence to: Dr. B.A. Finegan, Department of Anaesthesia, Foothills Hospital at the University of Calgary, 1403 29th St NW, Calgary, Alberta, T2N 279.
Adverse reactions have been reported following intravenous regional anaesthesia, related to the systcmic toxicity of the local anaesthetic (LA) agent used. These usually have been noted following inadvertent or early release of the toumiquet, or because of equipment malfunction. ${ }^{1-3}$ However, leak of contrast material during injection, with an intact toumiquet has also been noted, ${ }^{4}$ and there have been at lcast two case reports of severe reactions in these circumstances due to alleged leakage of LA. ${ }^{5,6}$ The mechanism for such leakage was presumed to be the development of excess pressure in the venous system of the isolated limb, although this has never been measured. Given the compliance and capacity of the venous system such an hypothesis seems unlikely and we therefore decided to investigate the pressure/volume relationship in an experimental situation analogous to performing an intravenous block.

\section{Methods}

Ten healthy members of the Department of Anaesthesia, five male, five female, of known height and weight, having given informed consent were studied. Following supine blood pressure estimation and under continuous ECG monitoring, a $22 \mathrm{ga}$ intravenous cannula ("Quick-cath" or Jelco) was inserted into a vein on the dorsum of one hand under LA infiltration (lidocaine one per cent). This cannula was connected to an extension set with two way tap (Pharmaseal 75052). A 20 ga intravenous cannula ("Quick-cath") was inserted, under LA as above, into a large ante-cubital vein in the same arm. This second cannula was connected via a monitoring kit (Sorenson MK4-07 BNVF) to a Bentley Trentec transducer (Model 800) the output of which was recorded on a Grass recorder (Model 7D Polygraph). A pneumatic double-cuffed toumiquet ("Inflato-Matic" 2000 , Zimmer) was affixed to the test limb about the 


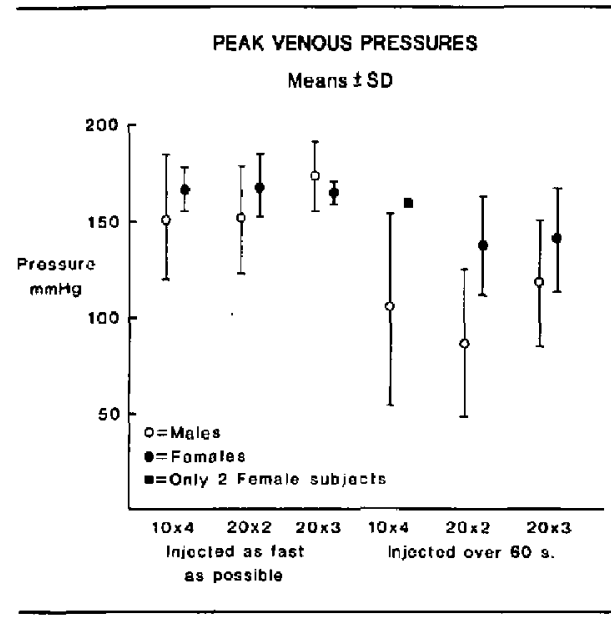

FIGURE 1 Peak venous pressures.

elbow. Its competence and accuracy was tested before each series of observations.

After limb elevation for three minutes (to exsanguinate the $\mathrm{arm}^{2}$ ) the cuff was inflated $150 \mathrm{mmHg}$ above the measured systolic blood pressure. Saline was injected via the distal cannula and the pressure response recorded. On development of a steady state, after injection was complete, the cuff was deflated and the limb elevated again for three minutes before reinflating the cuff prior to the next injection. To determine the volume/time relationship, injections of $10 \mathrm{ml} \times 4,20 \mathrm{ml} \times 2$ and $20 \mathrm{ml}$ $\times 3$ were made as quickly as possible (about 25 seconds) and then repeated over approximately 60 seconds, so that each subject received a total of six injections. All injections were performed by one investigator.

\section{Results}

The results in both groups are shown in Figure 1. In one female subject, the $20 \mathrm{ml} \times 3$ injections were omitted due to extreme discomfort during the $20 \mathrm{ml}$ $\times 2$ series, also the $10 \mathrm{ml} \times 4$ injections over 60 seconds were abandoned during the project as pressure changes were not significantly different from the $20 \mathrm{ml} \times 2$ injections over 60 seconds.

As shown in Figure 1 there was wide variation in mean peak venous pressures between individuals, although the overall patterns are similar between males and females. The mean cuff pressure in males

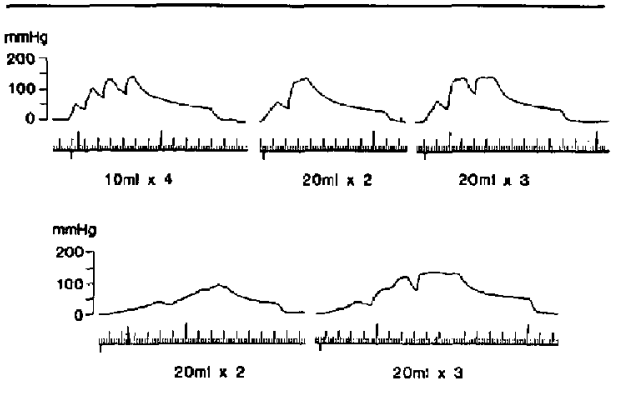

FGURE 2 Venous pressure traces: the upper three traces show the effects of injection as fast as possible, the lower two traces show the effects of injection over one minute.

was $276 \mathrm{mmHg}$ and in females was $263 \mathrm{mmHg}$. The initial venous pressure in all individuals was between 0 and $20 \mathrm{mmHg}$. The highest recorded venous pressure in our subjects was $190 \mathrm{mmHg}$ noted in one female and two males. The resting venous pressure after infusion but before the cuff was deflated varied from 20 to $50 \mathrm{mmHg}$ (means: males 28, females 34).

Figure 2 shows a typical venous pressure trace with rapid rise in pressure reaching a plateau at a level well below cuff pressure. It proved impossible to achieve a pressure greater than this plateau with the volumes injected. The slower injections followed a similar trend but only reach the plateau phase later in the injection period.

\section{Discussion}

The venous system is characterized by thin walled vessels which adapt to alterations in volume by changing from an elliptical to a circular profile. Thus large volumes can be accommodated with little increase in venous pressure. Once the cross sectional profile is circular, however, a vein is less distensible than an artery. ${ }^{\text {? }}$ In the isolated and exsanguinated limb, the capacity of the venous system would not be expected to be exceeded by the volumes of fluid administered in clincal practice. Leakage of contrast material has been radiographically observed in these circumstances, ${ }^{5}$ however, and high venous pressure in the isolated limb suggested as the cause.

Venous pressure levels in our study never approached cuff pressure and were relatively little influenced by speed of infusion. The development of a plateau was surprising but was most likely due 
to redistribution of the injectate to the deep veins and capillaries by retrograde valve opening. Other possible mechanisms include flow through intraosseus venous channels and nutrient vessels. The use of hyperosmolar contrast medium, noted in a report, ${ }^{5}$ apparently injected by a mechanical device does not necessarily reflect the clinical use of intravenous regional anaesthesia, as the mode of injection is not analogous to the manual method usually employed and the nature of the contrast medium could affect venous tone. This report also describes a case of acute bupivacaine toxicity which occurred with an intact cuff following the injection of $80 \mathrm{ml}$ of solution in 30 seconds. In the present study we were unable to achieve this rate by manual injection.

Clearly the isolated arm has a finite compliance and infusion of sufficient volume of solution, injection at a sufficiently high rate ${ }^{5}$ (especially via a mechanical device designed to infuse contrast medium) or injection at the ante-cubital fossa ${ }^{4}$ can generate a venous pressure greater than that in the occluding cuff. This would allow escape of LA into the systemic circulation. However, our study shows that when normal clinical volumes are injected at reasonable rates via veins on the dorsum of the hand there is no reason to expect venous pressure in the isolated limb to even approach, let alone exceed, indicated cuff pressure. Due to individual variation in arm morphology and cuff design it is not possible to state that the pressure exerted on the deep veins is the pressure indicated by the cuff manometer. A minimum cuff width to length ratio of $1: 3$ and an inflatable bladder which completely encircles the arm have been advocated to effectively transmit pressure from the periphery to the core of the limb, and allow accurate measurement of blood pressure. ${ }^{10}$ The two inflatable bladders of the cuff used in the present study were $65 \mathrm{~cm}$ in length, $5 \mathrm{~cm}$ each in width and fully encircled the arms of all the subjects. As both cuffs were inflated the recommended criteria were satisfied. However, the pressures measured at the ante-cubital fossa in this study (190 $\mathrm{mmHg}$ maximum) might give rise to leakage via the deep venous system if narrower or inadequately inflated cuffs are used.

An alternative explanation for the measurable concentrations of LA that have been detected in the systemic circulation in patients with intact toumiquets ${ }^{8}$ lies in the observation and investigation of "toumiquet coze", the dark blood that is often seen to ooze into wounds which are distal to a tourniquet. Furlow ${ }^{9}$ has suggested that the bleeding derives from intraosseous medullary canals leaking into the exsanguinated limb via nutrient vessels, thus avoiding toumiquet pressure. If this mechanism allows venous blood to travel through the medullary cavity with a pressure difference of perhaps $10-20 \mathrm{mmHg}$, it would seem possible for leakage to occur in the opposite direction under the $100-150 \mathrm{mmHg}$ pressure difference we have demonstrated as in a Bier block, thus allowing small but measurable amounts of LA into the systemic circulation.

From our results it appears that there is little risk of leakage of significant amounts of LA past an intact tourniquet provided that:

(a) The site of injection is as distal as possible to the occluding cuff, ideally on the dorsum of the hand.

(b) The volume of injectate is not excessive; we would suggest that no more than $60 \mathrm{ml}$ is ever necessary in the upper limb.

(c) The rate of injection is not excessive; if injection is made manually through a small cannula, it is difficult to see how an excessive rate could be reached; however, we would suggest that injection should take place over no less than one minute.

We would also suggest that the least toxic LA agent should be chosen unless contraindicated for some other reason, thus reducing the hazard of LA toxicity should inadvertent cuff failure occur.

\section{References}

1 Aronson $H B$, Vaiashsky $E$. Inadvertent toumiquet release five minutes after intravenous regional bupivacaine. Anaesthesia 1980; 35: 1208-10.

2 Fleming SA. Safety and usefulness of intravenous regional anaesthesia. Acta Anaesthesiol Scand Suppl 1969; XXXVI: 21-5.

3 Heath $M L$. Deaths after intravenous regional anaesthesia. Br Med J 1982; 285: 913-4.

4 Raj PP, Garcia CE, Burleson JW, Jenkins MT. The site of action of intravenous regional anaesthesia. Anaesth Analg 1972; $51 ; 776-86$.

5 Rosenberg PH, Kalso EA, Touminen MK, Linden $H B$. Acute bupivacaine toxicity as a result of venous leakage under the toumiquet cuff during a Bier block. Anaesthesiology 1983; 58: 95-8. 
6 Davies JAH, Gill SS, Weber JCP. Intravenous tegional analgesia using bupivacaine. Anaesthesia 1981; 36: 331 .

7 Keele CA, Neil E. Samson Wright's Applied Physio]ogy, Twelfth Edition. Oxford University Press. 1971, pp. 87-9.

8 Kalso E, Tuominen MK, Rosenberg PH, Alila $A$. Bupivacaine blood levels after intravenous regional anaesthesia of the arm. Regional Anaesthesia 1980; 5: 81-4.

9 Furlow $L T J r$. Cause and prevention of tourniquet ooze. Surg Gynecol Obstet 1971; 132: 1069-72.

10 Steinfeld $L$, Alexander $H$, Cohen $M L$. Updating sphygmomanometry. Am J Cardiology 1974; 33: $107-10$.

\section{Résumé}

Les changements de pression veineuse ons été mesurés dans une veine antécubitale d'un membre isolé pendant l'administration de salin par une veine du dos de la main. Bien que la perte de produit de contraste ait déjd été démontrée radiologiquement dans ces circonstances, la compliance du système veineux du membre isolé et exsanguiné n' a jamais été étudiée. Chez cinq hommes et cinq femmes volontaires, on a procédé après l' exsanguination et l' isolation du membre supérieur à l' injection manuelle de 40 a 60 ml de salin. Les changements de pression dans la veine antécubitale on été enregistrés continuellement pendant et après l'injection, jusqu'à l'obtention d' un état stable.

Quoique les pressions décrites aient atteint $190 \mathrm{mmHg}$ précocément après le début de l' injection, elles tendaient généralement à se stabiliser avani la fin de l'injection et à rester constantes par la suite. La pression veineuse de repos à la fin de l' injection était entre 20 et $50 \mathrm{mmHg}$ pour tous les volontaires. Jamais la pression veineuse n'approcha la pression du garrot; donc, le mécanisme de fuite de liquide en présence d' un tourniquet intact ne s'explique pas par le développement d'une pression veineuse excédant celle du tourniquet. 